\title{
Patient care and physician conflicts of interests: the Hydra grows new heads but is any Hercules in sight?
}

\author{
Sanjeev Saksena
}

Published online: 10 February 2015

(C) Springer Science+Business Media New York 2015

The start of a new year always gives us another chance to reflect on what has been achieved and what needs to be still done. In 2014, the Journal has prospered and grown in scope and submissions. This has been a particularly successful year in bringing more participation in the direction of the Journal and broadening its leadership. We have continued to define a clearer vision and found new avenues to bring better and more diverse science to our readership. The online availability of the Journal has become more facile. New members have joined the editorial leadership, and a series of new initiatives have taken root. Their fruits will be apparent in due course.

It is becoming a tradition now for the inaugural editorial each year to address a complex, often vexing, and certainly challenging issue. This year, I have chosen to turn to the issues of physician/investigator conflicts of interest as new dimensions continue to evolve. These new developments challenge the daily performance of clinical care and, in turn, impact the clinical investigation environment. This issue first came to my notice just as I commenced my career as an independent investigator in 1980. A prescient editorial was published that year by Arnold Relman entitled the "medical-industrial complex" in which he famously noted "the most important health-care development of the day is the recent, relatively unheralded rise of a huge new industry that supplies health-care services for profit." This commentary catapulted this issue into the dead center of the health care debate, where it remains till today [1]. Clinical and interventional electrophysiology as a medical discipline was but a twinkle in some of our eyes at that time.

\section{S. Saksena $(\triangle)$}

Rutgers-Robert Wood Johnson Medical School, Piscataway and the Electrophysiology Research Foundation, 161 Washington Valley Road, Suite 201, Warren, NJ, USA

e-mail: cmenj@aol.com
Relman went on to comment "in this medical market, physicians must act as discerning purchasing agents for their patients and therefore should have no conflicting financial interests. Closer attention from the public and the profession, and careful study, are necessary to ensure that the 'medical-industrial complex' puts the interests of the public before those of its stockholders." Fifteen years later, I recall participation in another informal conversation about the health care industry with leaders in clinical electrophysiology. Now representing the government relations arm of the then North American Society of Pacing and Electrophysiology, and government relations editor for PACE, I off-handedly observed to my colleagues "that health care cannot just be a business." I was reminded (read chided) that I may have missed the fact the health care industry is just that, a business. I recall responding that it could not be run in the long-term as a business solely on financial principles. Almost a decade later, all of us revisited this issue in the era of stalled expansion of managed care and the telling rejection of such draconian management of health care access by the American patient.

Yet 35 years later, there is little doubt of the continuing relevance of the Relman commentary. Financial incentives (among others) that can influence health care professional performance in their duties have been widely identified. Codes of conduct have been established by professional societies and in the law. While too many to enumerate here, these issues now encompass all aspects of a medical professional's work and life experience. Starting in the 1980s with the regulation of industry support of continuing medical education, pharmacy committees at hospital with serious bottom line input from hospital management, research grants, medical consulting, lecture honoraria, and litigation consultation, the list continues to evolve. Importantly, transparency in these 
relationships has been adopted in most spheres of health care professional-industry interactions.

While the relationship with the for- profit medical industry has been the focus of this issue, physician incentive programs directed at monitoring and channeling direct clinical care are now evolving, growing new heads of this Hydra with each passing year. In 2015, a new development has taken firm root, financial incentives for "efficient" medical care and the use of electronic medical records. These incentives are being provided by not-for-profit governmental agencies, but are often given to for- profit hospitals, physician practice organizations, and even individual physicians. Going well beyond the Relman doctrine, in the age of electronic medical records and "big-data", this introduces new "incentives" that can change physician behavior driven by personal and institutional financial considerations and engender patient care concerns. For the practicing electrophysiologist, these have special concerns that I will highlight here. Key to this approach of behavior modification is the availability of necessary data from electronic medical records. To achieve this end, physicians are financially penalized now for not maintaining and reporting data from electronic medical records and are financially rewarded for doing so.

Electronic medical records and financial data now allow for monitoring and analysis of physician behavior and practice patterns. Hospitals now provide physician "dashboards" that define practice patterns and to a limited extent, short-term indices of quality of care such as in-hospital adverse events or 30-day readmission rates (Fig. 1). The availability of such data is clearly desirable in reviewing and improving quality of medical care by institution of better medical practices. How can this data become a patient care concern? One such avenue gaining momentum is the use of cost- reduction incentives for hospitals. Hospitals now receive financial incentives and can institute "physician gainsharing programs" to parcel out "bonuses" according to economic impact of an individual physicians' care relative to established metrices. A cardinal metrix is the average patient length of stay adjusted for "severity" indices. The relative weight of these components in a given metrix and its validation remains less than rigorously established by carefully constructed prospective studies.

For clinical electrophysiologists who provide a tertiary level of care, this poses special dilemmas. Arrhythmia management can be necessary emergently or subacutely, with timing of antiarrhythmic interventional procedures being dependent on staging in an overall cardiovascular treatment algorithm. All too often, this occurs later in a hospitalization. For more elective interventions, comorbid conditions can intrude and require careful management. In either instance, the pressure to avoid prolonging hospital stay is significant, and when coupled with financial incentives, can be a particularly toxic combination impacting optimal patient care. Among those that would speak favorably about targeted incentives to impact inpatient health care costs, the rejoinder would include the use of quality of care metrices to balance a rush to patient discharge. And why should this issue be particularly important for one subspeciality, as it can be a generic concern? And is it truly a new "conflict of interest"?

While clinical electrophysiologists may not be totally unique, they have often served as an early warning system
Fig. 1 Individual physician inpatient clinical activity at one hospital during a defined analysis period is shown using a dashboard format. The gauge needle provides guidance as to how the individual physician data compare to a comparator standard. Only a few selected fields are shown to provide a conceptual rendering of this data report. Green reflects a favorable performance while orange or red exceed the comparator standard
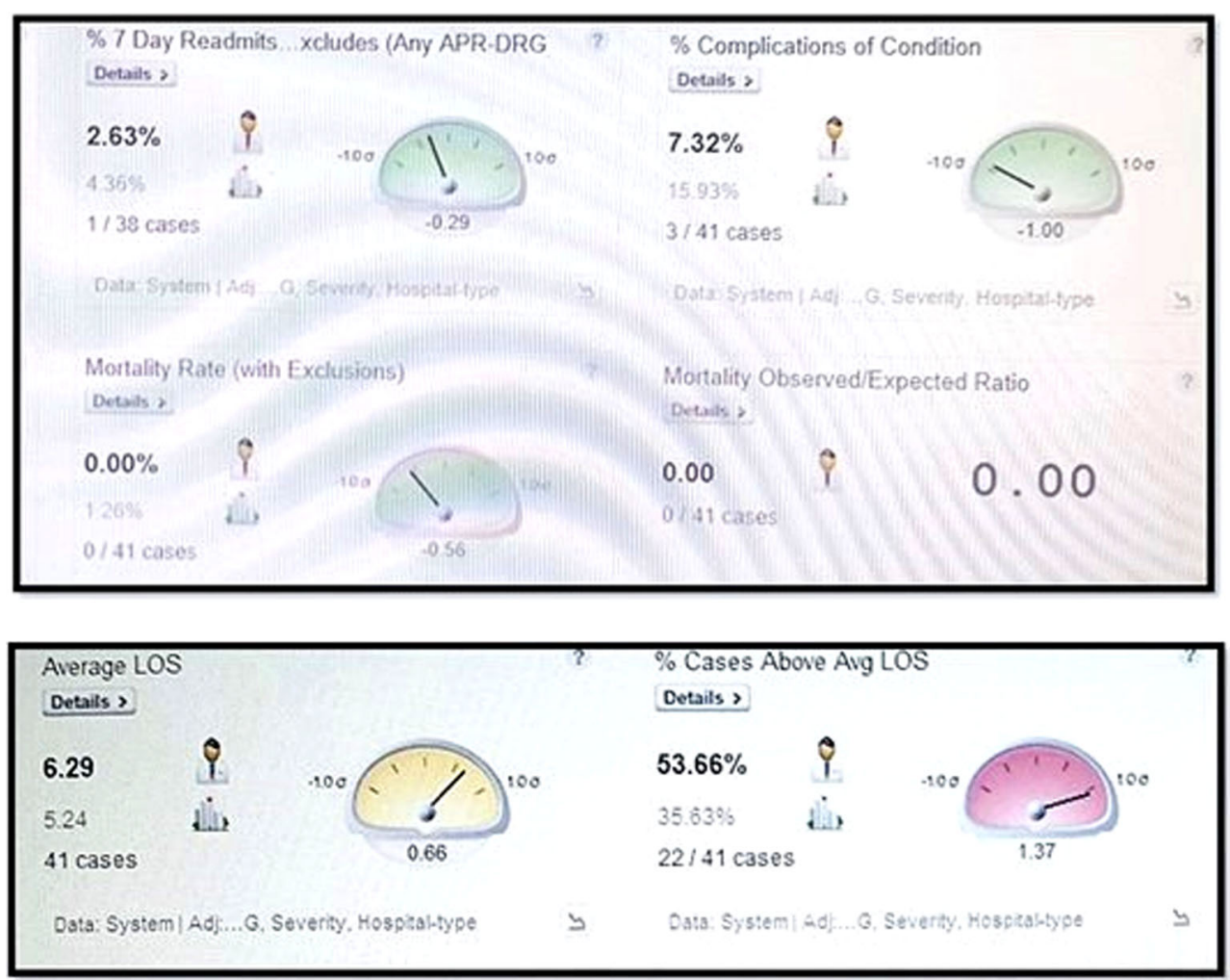
for specialty care, as those involved in such matters on behalf of this specialty have experienced. This may be another such instance. While gaming the metrices in use is entirely feasible (and practiced to a larger degree than recognized), this does not serve the needs of better patient care. One cogent example can speak to this point. Recently, Pathak and coworkers reported the results of the ARREST-AF study [2]. They noted that the long-term outcome of catheter ablation in atrial fibrillation is seriously impacted by effectiveness of comorbidity management. Excellent control of risk factors such as diabetes, hypertension, sleep apnea, smoking, alcohol excess, and other conditions reduced the need for repeated ablative procedures during longer-term follow-up. While the control group was not randomized and comprised patients refusing risk factor management participation in a dedicated clinic, the study nevertheless showed gains in both patient care, and by inference health care costs in the long-term by initiating such a program. Such programs require coordination of care best done in the hospital setting to initiate the patient into longitudinal well-managed health care in the outpatient setting. Current short-term rewards for "efficient" patient care fail to recognize the reality of longer-term care for patients with increasing comorbidities that underpin arrhythmia surges, and are an essential component of management. In this commentator's opinion, it is such care should be funded and "rewarded." The arrhythmia community needs to strive to highlight the coordination needed in the health care team for optimal care and the time needed to achieve it, rather than focus on volume and rapid patient processing. In doing this, the electrophysiology community will again take a leadership role in highlighting the particular needs for disease-driven specialty care.

The ever present retort for new health care programs is the spiraling costs of health care. Another relevant vignette. In 1999, our non-profit foundation initiated a project for expanding cardiac rhythm programs and implantable defibrillator (ICD) availability in India. ICDs were provided at cost, in those days about $\$ 5000$. Noting the postoperative hospital stay lasting several days, the US administrator of this program commented on the potential cost savings of 24-hour discharge policies. In response, the physician- in- charge pointed out that in the absence of third party reimbursement for ICDs, patients assumed the full costs of care and that the ICD was the main element of overall cost. He also noted that hospital stay costs were rather minor compared to that component, and that device infection and complications rates would have far larger clinical and economic impact for the patient.

The pertinent message for rising health care costs is simply this. The spiraling infrastructure and overhead costs of Western health care systems imposed by unaffordable and expensive regulations require far more scrutiny than compromises in direct patient care delivery. The impact of electronic medical records and algorithmic care systems on direct patient care delivery by medical professionals is yet another current case in point.
Patients are the first to notice the loss of professional face time, with an increase in algorithmically mandated testing and record- keeping time. Incentive payments and fines based on electronic medical recordkeeping performance measures have been instituted by the Center for Medicare Services. These may instead serve incentivize electronic record management, and actually further reduce direct patient care time [3]. Physician incentives that could lead to deleterious effects on direct patient care and adequate patient- physician interaction serve to lead us in a contrary direction.

Eleven years after his seminal warning, Relman revisited the changes in the US health care environment [4]. Recognizing new trends of market-driven health care, commercialization of voluntary hospitals, and medical practice being conducted as a competitive business, he asked physicians to consider divesting themselves of all such ties that can be construed as "conflicts of interest." Presciently, he spoke of the threat to the "morale of physicians and their social contract". A quarter of a century later, it is no longer the health care industry but governmental health care initiatives that pose a risk to this social contract. In fact, as the majority of physicians in the USA become corporate employees, this threat is magnified, not reduced. While independent practitioners may resist corporate guidance on behalf of their patients, this resistance easily may dissolve in the face of the employee-employer relationship. This threat is steadily becoming a reality in many health care systems globally.

The loss of physician morale is a clear and current threat, and the social contract of the physician with the patient is under siege as never before. This commentary can add little but more reiteration of the importance of the ongoing battle for the soul of the medical professional. We can and must now recognize that the Hydra that constitutes the "conflicts of interest" in a health care professional's existence continues to grow new heads. Like Hercules, we search for a "golden sword" to remove those that exist and prevent their proliferation. Protection of innovative direct patient care must remain at its heart.

In 2015, all of us at JICE hope to continue our pursuit of excellence in the service of our patients and our readership. At this time, we also recognize with gratitude those who labor unstintingly on behalf of the Journal, our esteemed editorial board and our pantheon of many reviewers. Without their efforts, we could not aspire to scientific excellence. Our editorial team at Springer led by Ms. Lisa Aquilino continues its fine work in executing the many tasks involved in producing a fine journal from beginning to end.

One additional New Year resolution for this commentator is to direct my "incentive" for "efficient medical care" to the Heart Rhythm Society. Personally, lacking a "golden sword," I wish to sidestep one more Hydra head in delivering better patient care this year.

Sanjeev Saksena MD

Editor- in- Chief

Respectfully submitted for the editors. 


\section{References}

1. Relman, A. S. (1980). The new medical-industrial complex. New England Journal of Medicine, 303, 963-970.

2. Pathak, R. K., Middeldorp, M. A., Lau, D. A., et al. (2014). Aggressive risk factor reduction study for atrial fibrillation and implications for the outcome of ablation: the ARREST-AF cohort study. Journal of the American College of Cardiology, 64(21), 2222-2231.

3. Gottlieb S (2014). Obamacare's threat to private practice. The payment system is forcing doctors to sell out to hospitals. The trend, and the law, will be unstoppable without reform. Wall Street Journal December 7.

4. Relman, A. S. (1991). The health care industry: where is it taking us? New England Journal of Medicine, 325, 854-859. 